\title{
EVALUATIONSVERFAHREN ALS TEIL DES UNIVERSITÄREN QUALITÄTSMANAGEMENTS - ERFAHRUNGEN AUS DER PRAXIS DER TU GRAZ
}

\author{
GERALD GABERSCIK UND MANUELA BERNER
}

DOI: 10.22163/fteval.2019.456

\section{PRÄAMBEL}

$\mathrm{M}$ it der (Rück)Übertragung von Autonomie an die österreichischen Universitäten und der damit verbundenen Rechenschaftspflicht mussten die Universitäten, ergänzend zu den verpflichtenden Quality Audits der externen Qualitätssicherung, ein internes Qualitätsmanagement (OM) aufbauen. Jene, die wie z.B. die TU Graz frühzeitig damit begannen, haben nunmehr einen Erfahrungshintergrund von ca. fünfzehn Jahren. Ein ausreichend großer Zeitraum, um Unterschiedliches auszuprobieren, zu bewerten und zu optimieren. Nachfolgend wird dargestellt, wie die TU Graz Evaluationen von Fachbereichen und Fakultäten als ein Werkzeug in ihr internes OM integriert hat, welche Vorgangsweise gewählt wurde und wie sich diese bisher bewährte, welche Wirkungen erzielt wurden sowie welche Randbedingungen Grenzen setzen. Abgerundet wird dieser Erfahrungsbericht durch eine kurze Analyse des Aufwands.

\section{EINLEITUNG}

Beim Aufbau des internen OM, das die TU Graz bereits im Jahr 2011 als erste österreichische Universität und noch vor dem in Krafttreten des HS-OSG ${ }^{1}$ zertifizieren ließ, wurde darauf Rücksicht genommen, dass sich die Bestimmung der Qualität ${ }^{2}$ der Kernaufgaben einer Universität - Forschung und Lehre - einer direkten Messung „in Metern und Sekunden” weitgehend entziehen. Als einzig sinnvoll erscheinendes und im wissenschaftlichen Bereich auch anerkanntes Verfahren zu Approximierung der Qualität von Forschung und Lehre wurden Evaluierungsverfahren identifiziert und als wesentlicher Bestandteil ins OM-System integriert. Es wurde ein Zyklus festgelegt, der über mehrere Jahre hinweg Evaluierungen von Fachbereichen und Fakultäten mit internen und externen Audits synchronisiert und zusätzlich mit den Perioden der Entwicklungsplanerstellung sowie den Leistungsvereinbarungen der Universität mit dem Bundesministerium abgestimmt ist. Ergänzend wird noch darauf Rücksicht genommen, dass die TU Graz mehrere interuniversitäre Kooperationen betreibt, die sowohl kooperative Forschung als auch gemeinsame Studienangebote umfassen ${ }^{3}$ und eine Abstimmung mit den Partnerinstitutionen erfordern.

Vor dem Start des aktuellen Durchgangs des Evaluierungszyklus wurden zwei unterschiedliche Vorgangsweisen analysiert und hinsichtlich Anwendbarkeit, Nutzen und Akzeptanz verglichen: Eine Option betraf den Einsatz von Kennzahlen, um einen Überblick über den Evaluierungsgegenstand zu gewinnen, die andere war die Durchführung eines Peer Review-Verfahrens, um qualitative Fragestellungen zu beantworten. Jede dieser Alternativen hat Vor- und Nachteile; so können Evaluationsmethoden, die sich ausschließlich auf Indikatoren stützen zwar rasch und mit geringem Aufwand realisiert werden, allerdings ist die Treffsicherheit bei der vorliegenden komplexen Sachlage nicht besonders ausgeprägt. Peer Review Verfahren wiederum werden der Komplexität besser gerecht und genießen im Wissenschaftsbereich eine recht hohe Akzeptanz, dennoch bestehen, neben dem relativ großen Aufwand, auch Nachteile, wie beispielsweise die Subjektivität infolge des spezifischen Erfahrungshintergrunds der agierenden Peers und die bekannte „Mainstreambevorzugung".

Unabhängig davon, für welche Methode man sich entscheidet, so ist jedenfalls sicherzustellen, dass die Evaluation die vier grundlegenden Ei-

HS-QSG, Bundesgesetz über die externe Qualitätssicherung im Hochschulwesen und die Agentur für Qualitätssicherung und Akkreditierung Austria, BGBI. I Nr. 74/2011, i.d.g.F.

2 Qualität $=$ Relation zwischen realisierter und geforderter Beschaffenheit, Walter Geiger / Willi Kotte, Handbuch Qualität, 5. Auflage, Vieweg, Wiesbaden, 2008, S 68

3 Beispielhaft sei hier auf NAWI Graz, eine Kooperation der KF Universität Graz und der TU Graz, oder das Toningenieurstudium, eine Kooperation der Kunst Uni Graz und der TU Graz, verwiesen. 
genschaften "Nützlichkeit - Durchführbarkeit - Fairness - Genauigkeit", aufweist, wie sie in den Evaluationsstandards der DeGEval ${ }^{4}$ detailliert beschrieben sind. Damit kann man weitestgehend sicherstellen, dass das gewählte Verfahren neutral gegenüber diversen inhärenten Eigenschaften des Evaluationsgegenstandes und Randbedingungen ist. Trotz aller Sorgfalt kommt man jedoch nicht um das grundsätzliche Spannungsfeld „Aktualität - Reichhaltigkeit - Vergleichbarkeit - Validität - Legitimität" der zugrundeliegenden Fakten und damit der bei der Evaluierung produzierten Information herum ${ }^{5}$. Darüber hinaus sind auch die Ziele, die durch die Evaluation erreicht werden sollen von Bedeutung bei der Auswahl der Methode.

Unter Berücksichtigung möglichst vieler Aspekte wurden zur fachund sachgerechten Beurteilung von Fachbereichen und Fakultäten Informed Peer Reviews für die angestrebten summativen Evaluierungen mit einem prospektiven Anteil als das am besten geeignete Verfahren ausgewählt.

\section{MOTIVATION UND ZIELE}

Die Entwicklungsmodelle in der Wissenschaftstheorie gehen von vier möglichen Szenarien aus. Veränderungen können demnach moderat (linear), exponentiell, stufig oder chaotisch ablaufen ${ }^{6}$. Entwicklungen entsprechend der drei ersten Modelle können, je nach Intention, von Universitätsleitungen angestrebt werden. Das vierte Modell wird wohl kaum gezielt verfolgt werden. Inwieweit die gesteckten Entwicklungsziele umsetzbar und welche Resultate letztlich zu erzielen sind, hängt aber von einer Vielzahl von Faktoren und Randbedingungen ab. Eine der wohl wichtigsten Einflussgrößen im österreichischen Universitätsbereich ist die periodisch zwischen der Universität und dem zuständigen Ministerium geschlossene Leistungsvereinbarung und der vorgelagerte Prozess der Entwicklungsplanerstellung. Um nicht in das vierte, ungewünschte Entwicklungsszenario zu verfallen, sind an der TU Graz die Abläufe, äquivalent zum bekannten TOM-Ansatz ${ }^{7}$, in einem „Drei Schalenmodell” entsprechend dem PDCA-Zyklus ${ }^{8}$ strukturiert. Die äußerste Schale wird dabei vom übergeordneten Ministerium, die mittlere von der Universitätsleitung und die innerste von den Fakultäten gebildet. Ein „Werkzeug” unter mehreren in diesem "Drei Schalenmodell" ist die Evaluation von Fakultäten mit der Motivation, die Weiterentwicklung geregelt voranzutreiben ${ }^{9}$.

Die Ziele des Rektorats bei den Fakultätsevaluierungen sind leicht nachvollziehbar. Es soll damit ein Prozess der Entwicklungsplanung in- nerhalb der betreffenden Fakultät unterstützt und das Selbstbild mit einer externen Sichtweise verglichen werden. (Hier erkennt man unschwer den Einfluss der Ziele auf die einzusetzende Evaluierungsmethode, denn nicht jede Methode beinhaltet eine Außensicht.) Darüber hinaus soll die Evaluierung den fakultätsinternen Kommunikationsfluss stimulieren und ausbauen. Und letztlich bilden die Ergebnisse aus der Evaluierung - der Peerbericht mit seinen Empfehlungen und die zugehörige Stellungnahme der Fakultät - eine wichtige Basis für Zukunftsentscheidungen des Rektorats. Für Fachbereichsevaluierungen gelten i.A. äquivalente Zielsetzungen, nur eben auf den Fachbereich bezogen, die ggf. noch um ein paar ergänzende Spezifika erweitert werden.

Da an der TU Graz, trotz der Herausforderung der großen Studierendenzahl bezogen auf die gegebenen Ressourcen, das humboldtsche Ideal der Einheit von Lehre und Forschung ${ }^{10}$ als weiterhin gültig erachtet wird und die universitätstypische, forschungsgeleitete Lehre einen fundamentalen Grundsatz darstellt, versteht es sich von selbst, dass bei einer Fakultätsevaluation ein gesamtheitlicher Ansatz zum Einsatz kommt. Es werden die Bereiche Forschung und Lehre immer gemeinsam betrachtet. Die Organisation und strukturelle Einbettung der Fakultät in der Universität, aber auch in der internationalen Scientific Community, ergänzen den Umfang zur einer umfassenden Gesamtevaluierung.

\section{VORBEREITUNG - BEGLEITUNG - NACHLAUF}

Vor dem Start des aktuellen Evaluierungsdurchgangs wurden die vorliegenden Erfahrungen mit der Durchführung von Peer Review Verfahren ${ }^{11}$, mit und ohne Begleitung durch eine Agentur, analysiert. Im Wesentlichen steht bei Verzicht auf eine Agentureinbindung dem Vorteil der Kostenreduktion ein erhöhter interner Organisationsaufwand gegenüber. Nicht unwesentlich sind auch die von einer guten Agentur geleistete Vorbereitung der Peers auf die österreichischen Randbedingungen und vor allem die Begleitung bei der Erstellung des Peerberichts nach dem Vorortbesuch. Die gute Kenntnis der österreichischen Situation und ein klar strukturierter, auf alle Evaluierungsfragen eingehender Bericht sind immens wichtig für die Zielerreichung.

Eine Kosten-/Nutzenabschätzung führte bei den gegebenen personellen Bedingungen dazu, dass es für die TU Graz sinnvoller ist, die Unterstützung einer Agentur in Anspruch zu nehmen. Nach dieser grundsätzlichen Festlegung wurde vom Rektorat die Evaluationsreihenfolge

4 DeGEval - Gesellschaft für Evaluation e.V. (2008) (Hg.): Standards für Evaluation, Erste Revision 2016 Mainz, 2017, ISBN 978-3-941569-06-5 oder http:// www.degeval.de/ (09.04.2019, 11:00)

$5 \quad$ Vgl. Jochen Gläser, Forschungsevaluation: Auf der Suche nach dem kleinsten Übel, Zentrum Technik und Gesellschaft,

TU Berlin, undatiert, vermutlich 2014

$6 \quad$ Vgl. Johann Götschl, Research Design: Methodology of Science and Research, Danube University Krems, Austria, January 14-15, 2012

7 TOM: Total-Quality-Management bezeichnet die durchgängige, fortwährende und alle Bereiche einer Organisation erfassende, aufzeichnende, sichtende, organisierende und kontrollierende Tätigkeit, die dazu dient, Qualität als Systemziel einzuführen und dauerhaft zu garantieren (nach wikipedia, 09.04.2019, 10:00)

8 PDCA-Zyklus: Demingkreis, richtiger Shewhart Cycle, beschreibt einen iterativen drei- bzw. vierphasigen Prozess für Lernen und Verbesserung, PDCA steht hierbei für das Englische Plan - Do - Check - Act

$9 \quad$ Vgl. Gerald Gaberscik, Andreas Drumel: Sammeln, strukturieren, bereitstellen, einsetzen, nachverfolgen - was braucht es und was bringt es im Qualitätsmanagement, Vortrag bei der 5. Internationale Tagung für Qualitätsmanagement und Qualitätsentwicklung im Hochschulbereich des QM-Netzwerks der österr. Universitäten, Graz, 12. Februar 2019

$10 \quad$ Vgl. Wilhelm von Humboldt, Skizze zur preußischen Hochschulpolitik, 1810

11 Vgl. Gerald Gaberscik, Manuela Berner, Andreas Drumel, Renate Euler, Das Qualitätsmanagementsystem weiterentwickeln - Vom Aufbau zur kontinuierlichen Verbesserung oder von den Herausforderungen des operativen Betriebs, Vortrag auf der Frühjahrstagung des AK Hochschule der DeGEval, 14.-15. Mai 2018, FOM Essen 
beschlossen, bei der besonders auf die spezifischen Situationen der Fakultäten und Fachbereiche Bedacht genommen wurde. So ist es z.B. zweckmäßig, die Gesamtevaluationen so anzusetzen, dass ein möglichst großer Veränderungsspielraum durch bevorstehende Neuberufungen gegeben ist, denn die fachlichen Festlegungen der auszuschreibenden Professuren bestimmen die Ausrichtung einer Fakultät oder eines Fachbereichs und damit die Möglichkeiten, neue Schwerpunkte zu setzen langfristig. Zusätzlich wurde auf die interuniversitären Kooperationen Rücksicht genommen, so dass in diesen Kooperationen engagierte Fakultäten oder Fachbereiche synchron an allen beteiligten Universitäten oder, wenn möglich, sogar gemeinsam evaluiert werden. Die festgelegte Evaluationsreihenfolge wurde den leitenden Personen der Fakultäten kommuniziert, damit diese frühzeitig davon Kenntnis haben.

Am Beginn einer Evaluierung steht auch die Auswahl der unterstützenden Agentur. Dabei ist es wichtig darauf zu achten, dass die Agentur flexibel genug ist, um die von der Universität definierten Ziele nicht nur zu akzeptieren, sondern sie zu übernehmen und mitzutragen. Selbstverständlich spielen auch die Kosten eine Rolle, aber eine vertrauensvolle Zusammenarbeit zwischen der Universität und der Agentur ist ungleich bedeutender. Hilfreich ist bei einem Evaluationsdurchgang, mit einer Abfolge von Verfahren, auch eine große Konstanz in der Begleitung, denn damit wird sichergestellt, dass die Erfahrungen aus der Zusammenarbeit nachhaltig genutzt werden können. Die TU Graz hat, nach einem breiten Screening und unter Einbeziehung der vorliegenden Erfahrungen, die Agentur für Qualitätssicherung und Akkreditierung Austria (A0 Austria) für die Begleitung des laufenden Evaluationsdurchgangs ausgewählt und entsprechende Verträge geschlossen.

Vor dem Start jeder einzelnen Fakultätsevaluierung - und nur auf diese wird im Folgenden weiter eingegangen - werden, im Sinne des PDCA-Zyklus, die Grundzüge mit den Erfahrungen aus dem Vorverfahren gegengecheckt um ggf. Verbesserungen einfließen lassen zu können. Erst danach beginnt die Kommunikation mit der ausgewählten Fakultät. Als definierter Anfangspunkt wird ein (internes) Startmeeting vom Rektor einberufen. Der Teilnehmerlnnenkreis umfasst dabei neben dem Rektor nicht nur die Fakultätsleitung und die die Evaluation begleitendenden Personen aus Stabsstellen, sondern es wird immer auch eine Person beigezogen, die in einem kürzlich abgeschlossenen Evaluierungsverfahren einE wichtigeR Akteurln war. Durch diese Zusammensetzung werden einerseits die Bedeutung der Gesamtevaluierung klar herausgestrichen, andererseits aber auch die angebotenen Hilfestellungen aufgezeigt und eine Brücke zu ErfahrungsträgerInnen außerhalb der ausgewählten Fakultät und der Administration geschlagen. Im Startmeeting werden die Ziele der Evaluierung dargelegt, der prinzipielle Ablauf erläutert und der grobe Zeitplan kommuniziert. Gleichfalls weist der Rektor darauf hin, dass eine zusätzliche Ressourcenbereitstellung zur Abdeckung eines Teils des Aufwands erfolgt. Auch werden bereits erste Erfahrungen über den erforderlichen Aufwand, erfolgreiche Vorgangsweisen und erzielten Nutzen weitergegeben. (Als ein Beispiel dafür sei die bewährte organisatorische Struktur innerhalb der Fakultät angeführt. Es hat sich he- rausgestellt, dass es für die Erstellung des Selbstevaluierungsberichts und die Abwicklung des Vorortbesuchs günstig ist, ein relativ kleines Kernteam und ein breit aufgestelltes, alle Kurien ${ }^{12}$ umfassendes, Vorbereitungsteam zu installieren. Im ersten werden die Entwürfe vorbereitet, die dann im großen Team diskutiert und verbessert werden können. Das Vorbereitungsteam stellt auch einen „guten Fundus" für GesprächspartnerInnen beim Vor-Ort-Besuch dar, denn seine Mitglieder sind, durch die Mitarbeit in der Vorphase, mit der Sache wirklich vertraut.) Das Startmeeting wird mit einem Protokoll, in dem festgehalten ist, wer was bis wann zu erledigen hat, zusammengefasst.

Ebenfalls in einer sehr frühen Phase wird eine Informationsveranstaltung fixiert, bei der der Rektor die Hintergründe, die Ziele und den Ablauf der Gesamtevaluierung an die Fakultätsangehörigen kommuniziert und innen Rede und Antwort steht. Durch diese umfangreiche Kommunikation wird verhindert, dass Ängste entstehen, Gerüchte aufkommen oder ggf. vorliegende Abwehrhaltungen gestärkt werden.

Denselben Zielen sowie auch dem Kennenlernen und der Feinabstimmung des Evaluierungsleitfadens dient das erste Arbeitsmeeting mit der Agentur. Hierbei werden die Aufgabenverteilung, der Zeitplan sowie die Vorgangsweise bei der Peerauswahl etc. mit der Fakultätsleitung detailliert besprochen.

Bevor mit der Arbeit am Selbstevaluierungsbericht begonnen werden kann, müssen das Kernteam und das Vorbereitungsteam zusammengestellt werden, dabei kann von der Fakultät auf die gesammelten Erfahrungen der Stabstelle Qualitätswesen an der TU Graz zurückgegriffen werden. Auch die Brückenbildung zu Akteurlnnen von abgeschlossenen Evaluierungen ist hilfreich für diesen Vorgang und allen folgenden Aktionen.

Einer der entscheidensten „äußeren“ Faktoren für den Erfolg einer Evaluierung sind die Peers. Deren Akzeptanz als „critical friends“, die ihre Erfahrungen zum Wohl der Evaluierten einbringen, ist die Grundvoraussetzung für die Bereitschaft Empfehlungen anzunehmen und umzusetzen. Damit kommt der Peerauswahl eine eminente Bedeutung zu. An der TU Graz wurden deshalb generelle Vorgaben für die Peerauswahl erarbeitet, die gemeinsam mit den Kriterien der Agentur sicherstellen, dass das Ziel einer hohen Akzeptanz der Peers möglichst gut erreicht wird.

Der von der Fakultät zu erstellende Selbstevaluierungsbericht ist sicherlich ein Schlüsseldokument im Peer-Review Verfahren. Er soll entlang des gut abgestimmten Evaluationsleitfadens, der auf den Zielen der Universitätsleitung basierend die Fragestellungen an die Peers enthält, den Ist-Stand der Fakultät für Externe nachvollziehbar darstellen (Grundlage der angestrebten summativen Evaluation). Eine ergänzende SWOTAnalyse ${ }^{13}$ soll darin den Ausgangspunkt für die geplante Entwicklung in den kommenden fünf bis sieben Jahren bilden (Grundlage für die prospektive Evaluation). Dabei ist die Balance zwischen umfassender Darlegung und überschaubarem Umfang besonders schwierig. Auch hierbei kann auf die im Haus vorliegenden Erfahrungen aufgebaut werden. Der Selbstevaluierungsbericht wird durch die Bereitstellung der TU-internen Datensammlung und -auswertung IBES ${ }^{14}$ ergänzt.

12 An Universitäten werden die internen „Stakeholder", die Gruppe der Professorlnnen, der Universitätsdozentlnnen und wissenschaftliche Mitarbeiterlnnen im Forschungs- und Lehrbetrieb, der Studierende und der Mitglieder des Allgemeinen Universitätspersonals als Kurien bezeichnet.

13 SWOT-Analyse (englisch für Strengths (Stärken), Weaknesses (Schwächen), Opportunities (Chancen) und Threats (Risiken)) ist ein Instrument der strategischen Planung; sie dient der Positionsbestimmung und der Weiterentwicklung

$14 \quad$ IBES = Input Balance und Ergebnissäulen. Dies ist eine Zusammenstellung von relevanten Daten aus den Bereichen Forschung, Lehre und Administration. Es werden auch dabei auch Input- und Output-Analysen vorgenommen und dem Mittelwert in der Betrachtungsgruppe gegenübergestellt. (vgl. Balancetorte und Effizienzspinne - ein Indikatorenset für Lehre, Forschung und Administration, Zeitschrift Hochschulmanagement HM, 4/2011, S 70 ff. ISSN $1860-3025$ Universitätsverlag Webler) 
Der nächste wichtige Meilenstein im Evaluationsablauf ist der Vorortbesuch. Eine gute Vorbereitung erleichtert die Durchführung erheblich. Schon bei der Auswahl der GesprächspartnerInnen und der organisatorischen Planung ist ein Zusammenwirken von Fakultät, Agentur und den involvierten universitären Stabsstellen sehr vorteilhaft, weil damit das Wissen um die Interna der Fakultät mit den Erfahrungen aus anderen Evaluierungsverfahren zusammenfließt.

Beim Vorortbesuch der Peers gilt es nicht nur die offenen Fragen der externen Expertlnnen zu klären, sondern auch eine vertrauensvolle Gesprächsbasis aufzubauen. Je besser es den Peers gelingt, die Fakultät in ihren Grundzügen und mit ihrer spezifischen Organisationskultur zu verstehen, desto hilfreicher werden die Empfehlungen im Peerbericht ausfallen können. Und je klarer der Bericht strukturiert auf die Evaluationsfragen eingeht, desto höher wird erfahrungsgemäß die Umsetzungswahrscheinlichkeit der gemachten Anregungen.

Endet mit der Übermittlung des Peerberichts der externe Teil der Evaluierung, so ist dies aber erst der Startpunkt für den universitätsinternen Nachlauf.

An der TU Graz wird der Peerbericht einerseits innerhalb der evaluierten Fakultät diskutiert und eine Stellungnahme dazu verfasst. Andererseits wird auch von Seite des Rektors der Bericht analysiert und die Empfehlungen werden auf Umsetzbarkeit hin durchleuchtet. In einem Aushandlungsprozess werden gemeinsam von Rektor und Fakultätsleitung ein Maßnahmenkatalog erstellt, der mit einer Zeitschiene versehen ist, und in dem die Verantwortlichkeiten für die Umsetzung festgelegt sind. Damit die vereinbarten Maßnahmen auch wirklich Realität werden, erfolgt ein periodisches Monitoring, das wiederum in die jährlichen Leistungsvereinbarungsgespräche zwischen Rektor und Fakultätsleitung einfließt.

\section{NUTZEN - AUSWIRKUNGEN - NICHT INTENDIERTE FOLGEN}

Ein wichtiger Nutzen einer Evaluierung liegt schon im Vorgang der Erstellung des Selbstevaluierungsberichts, denn es ist „ein Schritt heraus" aus dem operativen Tagesgeschäft unerlässlich, um die strategische Ausrichtung der Fakultät festlegen zu können. Auch wird der Selbstevaluierungsbericht nur gelingen, wenn bei seiner Erstellung eine gute Kooperation innerhalb der Fakultät erfolgt, und dabei wurden bereits oft neue Anknüpfungspunkte für die fachliche Zusammenarbeit erkannt. Auch die Erschließung der Erfahrungen der Peers, die mit ihrer Außensicht neue Blickwinkel einbringen, nützt den Beteiligten. Letztlich bringt ein derartiges Evaluierungsverfahren auch Nutzen für die Peers, denn auch sie profitieren fraglos von dem gewährten tiefgehenden Einblick in eine fachverwandte Fakultät einer anderen Universität.

Aus heutiger Sicht kann gesagt werden, dass die von der Universitätsleitung gesetzten Ziele der Evaluationen an der TU Graz sehr gut erreicht werden. Der Prozess der Entwicklungsplanung innerhalb der betreffenden Fakultät bzw. Fachbereiche wird fraglos unterstützt und das Selbstbild mit einer externen Sichtweise verglichen. Auch der fakultätsinterne Kommunikationsfluss wird merklich stimuliert und ausgebaut. Letztlich führen die Ergebnisse aus der Evaluierung - der Peerbericht mit seinen Empfehlungen und die zugehörige Stellungnahme der Fakultät zu einem Maßnahmenkatalog, der einer zielgerichteten Weiterentwicklung dient.
Eine nicht intendierte Auswirkung musste jedoch ebenfalls festgestellt werden. Die Peers neigen als FachkollegInnen der Evaluierten sehr leicht zu der Ansicht, dass für die betreffende Fakultät bzw. den Fachbereich viel mehr Ressourcen bereitgestellt werden sollten. Dies ist durchaus verständlich, da man annimmt, mit mehr Mittel mehr Möglichkeiten zu haben und mehr bzw. noch hochqualitativeren Output erreichen zu können. Allerdings setzen die realen Randbedingen hier enge Grenzen, so dass nunmehr die klare Vorgabe an die Peers besteht, bei Empfehlungen die Einschränkungen durch die bestehenden Möglichkeiten zu beachten.

\section{GRENZEN UND AUFWAND}

Bei den bisher durchgeführten Gesamtevaluierungen von Fakultäten oder Fachbereichen sind auch Grenzen zu Tage getreten. So wird von den Evaluierten immer eine Gratwanderung zwischen "gut dastehen wollen“ und dem „Ansprechen von Herausforderungen“ abverlangt. Ein wenig kann das entschärft werden, wenn die Vertraulichkeit von Selbstevaluierungsbericht und Peerbericht zugesichert wird. Gleichfalls hat die Kommunikation zwischen Rektor und Fakultät einerseits sowie Agentur und Fakultät andererseits einen großen Einfluss darauf, wie dies gelingt. Auch wie gut es gelingt, dass die Peers als "critical friends" anerkannt werden und damit eine weitgehende Akzeptanz der Empfehlungen erreicht werden kann, ist nicht immer gleich. Selbstverständlich wirkt jedenfalls mit, wie offen die wichtigsten Akteurlnnen, die "MeinungsbildnerInnen“ der Fakultät bzw. des Fachbereichs, für "fremde Ideen“ sind und wie hoch ihre Bereitschaft zur kritischen Selbstreflexion ist. Und letztlich gibt es Gestaltungsgrenzen, die gesetzlich festgelegt oder von der Ressourcenverfügbarkeit bestimmt werden.

Auch der Aufwand, der getrieben werden kann und soll, ist zu beachten. Neben den externen Kosten, die durchaus überschaubar im niederen fünfstelligen Eurobereich liegen, müssen die internen Aufwendungen beachtet werden, die in der Größenordnung des vier- bis achtfachen der externen Ausgaben liegen. Aus Sicht der TU Graz stimmt aber die Kosten-/Nutzenrelation.

\section{ZUSAMMENFASSUNG}

An der TU Graz stellen Evaluationen seit vielen Jahren einen integralen Bestandteil des Qualitätsmanagementsystems dar. Sie werden im Fall von Gesamtevaluierungen als Informed Peer Review, mit Unterstützung durch eine Agentur, ausgeführt. Wichtig sind dabei eine umfassende Kommunikation seitens der Universitätsleitung an die Evaluierten über die Motivation und die Ziele sowie auch eine gute Vorbereitung und Begleitung der Betroffenen. Erfolgskriterien sind ein kooperativer Vorgang bei der Erstellung des Selbstevaluierungsberichts durch die Fakultät bzw. den Fachbereich, eine hohe Akzeptanz der externen Peers als "critical friends", ein einvernehmliches Festlegen des Maßnahmenkatalogs auf Basis der Peerempfehlungen und ein konsequentes Monitoring der Umsetzung. Trotz allem gibt es Grenzen, die beachtet und akzeptiert werden müssen. Der Nutzen, der an der TU Graz aus den Evaluierungen bisher gezogen werden konnte, rechtfertigt den damit verbundenen Aufwand aber jedenfalls. 


\section{LITERATURVERZEICHNIS}

DeGEval - Gesellschaft für Evaluation e.V. (2008) (Hg.): Standards für Evaluation, Erste Revision 2016 Mainz, 2017, ISBN 978-3-941569-06-5 oder http://www.degeval.de/ (09.04.2019, 11:00)

Gaberscik, Gerald, Berner, Manuela, Drumel, Andreas, Euler, Renate: Das Qualitätsmanagementsystem weiterentwickeln - Vom Aufbau zur kontinuierlichen Verbesserung oder von den Herausforderungen des operativen Betriebs; Vortrag auf der Frühjahrstagung des AK Hochschule der DeGEval, 14. - 15. Mai 2018, FOM Essen

Gaberscik, Gerald, Drumel, Andreas: Sammeln, strukturieren, bereitstellen, einsetzen, nachverfolgen - was braucht es und was bringt es im Qualitätsmanagement; Vortrag bei der 5. Internationale Tagung für Qualitätsmanagement und Qualitätsentwicklung im Hochschulbereich des QM-Netzwerks der österr. Universitäten, Graz, 12. Februar 2019

Gaberscik, Gerald, Muhr, Hans Michael, Stelzer, Franz: Balancetorte und Effizienzspinne - ein Indikatorenset für Lehre, Forschung und Administration; Zeitschrift Hochschulmanagement HM, 4/2011, S 70 ff. ISSN 1860-3025 Universitätsverlag Webler

Geier, Manfred: Die Brüder Humboldt; Rowohlt Verlag, Reinbek bei Hamburg 2009, S. 267, ISBN 978-3-498-02511-3

Geiger, Walter, Kotte, Willi: Handbuch Qualität; 5. Auflage, Vieweg, Wiesbaden, 2008, S 68

Gläser, Jochen: Forschungsevaluation - Auf der Suche nach dem kleinsten Übel, Zentrum Technik und Gesellschaft; TU Berlin, undatiert, vermutlich 2014

Götschl, Johann: Research Design: Methodology of Science and Research; Danube University Krems, Austria, January 14-15, 2012

Humboldt, Wilhelm von: Skizze zur preußischen Hochschulpolitik, 1810

\section{AUTORiNNEN}

\section{DIPL.-ING. DR.TECHN. GERALD GABERSCIK}

Leitung Qualitätswesen, Technische Universität Graz

Rechbauerstrasse 12, $8010 \mathrm{Graz}$

E: gerald.gaberscik@tugraz.at

\section{MAG.RER.NAT. MANUELA BERNER}

Leitung Statistik und Berichtswesen, Technische Universität Graz

Rechbauerstrasse 12, 8010 Graz

E: manuela.berner@tugraz.at 